http://jmscr.igmpublication.org/home/ ISSN (e)-2347-176x ISSN (p) 2455-0450 crossref DOI: https://dx.doi.org/10.18535/jmscr/v8i8.03

\title{
Increasing Psycho-Social Stress among Doctors in COVID 19 Pandemic
}

\author{
Author \\ Dr Akhila Jose \\ Corresponding Author \\ Dr Akhila Jose
}

\begin{abstract}
Background: COVID19 has stricken the world alarmingly that even super powers are affected terrifically. The fight against the pandemic has become the centre stage of global activities. Doctors and other health workers are the frontline soldiers of all nations to shield the respective motherland from this pandemic. But they are the most vulnerable population who suffer their lives and there is an increasing psychosocial pressure among them. So this is an attempt to review the increasing psychosocial stress among doctors who work in the fore front of the war against COVID 19.

Methods: The key terms- "COVID19, "Pandemic", "psychosocial stress", "Health workers", Psychosocial stress" were searched in PubMed and Google Scholar. WHO official website was also searched for the latest updates. Newspaper sources were also utilised.

Results: The psychosocial stress among the doctors is increasing which even manifested as depressive and anxiety disorders. Very high risk of own health, social stigma of the community and fear of transmission to the family members has contributed to the increasing trend of psychosocial stress among the doctors.

Conclusion: Elaborate, coherent and coordinated measures should be taken by the government authorities to monitor the mental health and to prevent the social stigma arising in community towards our frontline warriors in this pandemic.
\end{abstract}

\section{Introduction}

In the last week of March 2020, the World Health Organization (WHO) declared COVID19 as Public Health Emergency of International Concern (PHEIC), which is the sixth PHEIC under the International Health Regulations after H1N1 Influenza (2009), Polio (2014), Ebola in West Africa (2014), Zika (2016), and Ebola in the Democratic Republic of Congo (2019). ${ }^{[1,2]}$ On February 11, 2020, the WHO has officially declared the COVID-19 as "pandemic" from the previous status of global health emergency. ${ }^{[4]}$
Healthcare workers (HCWs) play an inevitable` role at the front lines, providing care for patients. In the context of COVID-19 and during routine health services, they provide critical care to patients and ensure that infection prevention and control (IPC) measures are implemented and adhered to in healthcare facilities in order to limit healthcare-associated infections.

At least 96 doctors and 156 nurses have tested positive for COVID-19 across India as of April 22. As most of these infections were transmitted by patients in a hospital environment, at least 826 medical workers who came in contact with the 
infected personnel had to be quarantined and at least 20 hospitals had to be fully or partially closed. ${ }^{(7)}$

As of 8 April 2020, 22073 cases of COVID-19 among HCWs from 52 countries had been reported to WHO. However, at the present time, there is no systematic reporting of Health Care Workers COVID-19 infections to WHO and therefore this number probably under-represents the true number of COVID-19 health workers infections globally. To date, there are a limited number of publications and national situation reports that provide information on the number of HCW infections. For example, a publication from China CDC on 44672 confirmed cases as of 17 February 2020 indicated 1688 (3.8\%) infections were among Health Care Workers, including five deaths. In Italy, a situation report from 10 April 2020 reported 15314 infections among health care, representing $11 \%$ of all infections at that time. $^{(1)}$

In a pandemic, the number of patients requiring treatment increases significantly, placing strain on healthcare resources and on personnel alike. Additionally, doctors perceive a greater risk to self owing to their exposure to the patients adding further stress. Compounding to this stress is the shortage of personal protective equipment (PPE) that can arise during a pandemic. The perceived risk of infection is warranted: a meta-analysis of the occupational risk from the 2009 swine flu pandemic (influenza A (H1N1)) reports that the odds of healthcare personnel contracting the virus were twice those of comparison groups. This heightened risk for doctors and nurses might be due to their greater exposure to the respiratory secretions of patients. The increased risk of infection for the families of healthcare professionals on the front lineis another cause of concern .One way for doctors to mitigate infection risk to their families is through social distancing. However, although the protective benefits of social contact and support at times of stress are well demonstrated, social distancing deprives the individual of a crucial buffer against mental health difficulties precisely when they are at greater risk of stress. ${ }^{(3)}$

The great mental pressure are manifested as depressive disorders, and anxiety disorders which adversely affect their physical and mental condition and will significantly affect our health care system and also social stability as these people are the martyrs who risks their lives for the society. Hence there is a need to address these problems as we require a strong health care set up to cope up the situation. As the pandemic is affecting the economy of all nations adversely as the common people are affected worse and hence the increasing psycho social stress among doctors will indirectly affect the economy also.

\section{Discussion}

The alarming spread of infection leads to ameliorate the burden of work which poses a great need of more health care workers. Along with this fear of self risk and family members creates a great dilemmas among them when there is a threat of community spread. Inadequate protective equipment at the workplaces, social stigma, and also difficulty to maintain social distancing are some of the contributory factors which lead to increasing mental stress.

The psychosocial response of frontline workers during a pandemic is complex and incompletely understood. Studies regarding the 2003 SARS outbreak from Canada, Taiwan, and Hong Kong have discussed how the battle against SARS led to huge psychological morbidity amongst frontline HCPs. During the 2003 SARS outbreak in Taiwan, nurses working in the SARS unit suffered from more depressive symptoms and insomnia compared to those from non-SARS units and occurrence of psychiatric symptoms was associated with direct exposure SARS patient care. Even after three years of SARS outbreak in 2003, a significant number of the related hospital workers in Beijing, China experienced some PTSD. In the developing countries like India, where the health care system is already overburdened, surges of COVID-cases are likely 
to provoke acute anxiety, irritation and stress among doctors and nurses. $\mathrm{Li}$ etalin their nationwide study among HCPs working in fever clinics or treating COVID-19 patients during the 2019 Coronavirus outbreak showed that half of the responders identified at least depression and one-third reported insomnia whereas $14 \%$ of physicians and nearly $16 \%$ of nurses described moderate or severe depressive symptom. ${ }^{(5)}$

A cross-sectional study was done by Chatterjee, et al.: Impact of COVID-19 on doctors in West Bengal using an online semi-structured questionnaire and the survey has been done for 10 days. Data were collected on background characteristics, knowledge, attitude, and behavior of the respondents in a semi-structured pro forma, and psychiatric morbidity was measured by the Depression, Anxiety, and Stress Scale-21. A total of 152 complete responses have been received. The data were assessed using SPSS software. Results: Out of 152 study participants, 34.9\% were depressed and $39.5 \%$ and $32.9 \%$ were having anxiety and stress, respectively. Significant predictors for psychiatric morbidities were experience in health sector, duty hours, use of protective measures, and altruistic coping. Multivariable logistic regression showed most of the factors to be significantly associated with depression, anxiety, and stress level. ${ }^{(4)}$

A cross- sectional, web- based survey was conducted in February 2020 among healthcare workers during the COVID-19 pandemic by Que J, et al to investigate the prevalence of psychological problems in different healthcare workers (ie, physicians, medical residents, nurses, technicians and public health professionals). Psychological problems were assessed using the Generalized Anxiety Disorder Scale, Patient Health Questionnaire and Insomnia Severity Index. Logistic regression analyses were used to explore the factors that were associated with psychological problems. results The prevalence of symptoms of anxiety, depression, insomnia and the overall psychological problems in healthcare workers during the COVID-19 pandemic in China was $46.04 \%, 44.37 \%, 28.75 \%$ and $56.59 \%$, respectively. The prevalence of the overall psychological problems in physicians, medical residents, nurses, technicians and public health professionals was $60.35 \%, 50.82 \%, 62.02 \%$, $57.54 \%$ and $62.40 \%$, respectively. Compared with healthcare workers who did not participate in front- line work, front- line healthcare workers had a higher risk of anxiety, insomnia and overall psychological problems. $^{(6)}$

In a study published in Annals of Internal Medicine (C) 2020 American College of Physicians

Psychological Impact of the COVID-19 Pandemic on Health Care Workers in Singapore conducted from 19 February to 13 March 2020, health care workers from 2 major tertiary institutions in Singapore who was caring for patients with COVID-19 were invited to participate with a self-administered questionnaire. Of 500 invited health care workers, 470 (94\%) participated in the study; Sixty-eight (14.5\%) participants screened positive for anxiety, 42 (8.9\%) for depression, 31 (6.6\%) for stress, and 36 (7.7\%) for clinical concern of PTSD. The prevalence of anxiety was higher among nonmedical health care workers than medical personnel. Similarly, higher mean DASS-21 anxiety and stress subscale scores and higher IES$\mathrm{R}$ total and subscale scores were observed in nonmedical health care workers ${ }^{(2)}$.

\section{Conclusion}

In the awake of COVID19 pandemic, there is an increasing levels of depression, anxiety disorders, insomnia and various forms of psychosocial stress elements among the doctors and other health workers. This would significantly affect our health care quality and measures in fighting the pandemic. Hence it's an urgent need to address this issue and measures to be taken. Creating awareness among the public to cope up with prevailing stigmas and increasing the resources including protective equipment at the hospitals and treatment centres makes a significant change. Managing the working hours of the doctors in a 
healthy manner and more importance to be given to manage the mental health of every health care individual helps in a drastic change in the current issue of psychosocial stress among the doctors. This would help the individual, their family and also the society.

\section{Acknowledgement}

Here I thank all the supporting family members of all doctors and health workers worldwide who play a major role in fighting pandemic by giving all the mental and social support to the frontline warriors.

\section{Financial support and sponsorship: Nil.}

Conflicts of interest: There are no conflicts of interest.

\section{Reference}

1. World Health Organization. Coronavirus Disease 2019 (COVID- 19) Situation Report- 64. World Health Organization; 2020. Available from: https://www.who.int/docs/defaultsource/coronaviruse/situationreports/20200 324- sitrep- 64- covid- 19.pdf?sfvrsn=70 3b2c40_2.

2. Psychological Impact of the COVID-19 Pandemic on Health Care Workers in Singapore. Annals of Internal Medicine (C) 2020 American College of Physicians 1:6 April 2020.

3. Niall Galbraith, David Boyda, Danielle Mc Feeters, Tariq Hassan. The mental health of doctors during the COVID-19 pandemic. BJ Psych Bulletin (2020) Page 1 of 4, doi:10.1192/bjb.2020.44: Downloaded from https://www.cambridge.org/core.

4. Seshadri Sekhar Chatterjee, Ranjan Bhattacharyya, Sumita Bhattacharyya, Sukanya Gupta, Soumitra Das, Bejoy Bikram Banerjee. Attitude, practice, behavior, and mental health impact of COVID-19 on doctors.(C 2020 Indian
Journal of Psychiatry | Published by Wolters Kluwer-Medknow.

5. Souvik Dubey a, Payel Biswas, Ritwik Ghosh, Subhankar Chatterjee, Mahua Jana Dubey, Subham Chatterjee, Durjoy Lahiri, Carl J. Lavie. Psychosocial impact of COVID-19. Diabetes \& Metabolic Syndrome: Clinical Research \& Reviews 14 (2020) 779e788.

6. Jianyu Que, Le Shi,1 Jiahui Deng, Jiajia Liu, Li Zhang, 1, Suying Wu,Yimiao Gong, Weizhen Huang, Kai Yuan, Wei Yan, Yankun Sun, Maosheng Ran, Yanping Bao, Lin Lu. Psychological impact of the COVID-19 pandemic on healthcare workers: a cross- sectional study in China. General Psychiatry 2020; 33:e100259. doi:10.1136/gpsych-2020100259.

https://www.thehindu.com/data/howmany-doctors-and-nurses-have-testedpositive-for-coronavirus-inindia/article31410464.ece.

7. Sim K, Chua HC. The psychological impact of SARS: a matter of heart and mind. CMAJ 2004;170:811e2.

8. Wu P, Fang Y, Guan Z, Fan B, Kong J, Yao Z, Liu X, Fuller CJ, Susser E, Lu J, Hoven CW. The psychological impact of the SARS epidemic on hospital employees in China: exposure, risk perception, and altruistic acceptance of risk. Can J Psychiatr 2009;54:302e11.

9. Shigemura J, Ursano RJ, Morganstein JC, Kurosawa M, Benedek DM. Public responses to the novel 2019 coronavirus (2019-nCoV) in Japan: mental health consequences and target populations. Psychiatr Clin Neurosci2020;74:281e2.

10. Lima CKT, Carvalho PMM, Lima IAAS, Nunes JVAO, Saraiva JS, de Souza RI, et al. The emotional impact of Coronavirus 2019-nCoV (new Coronavirus disease). Psychiatr Res 2020;287:112915. 
11. Zandifar A, Badrfam R. Iranian mental health during the COVID-19 epidemic. Asian J Psychiatr2020;51:101990.

12. Antony MM, Bieling PJ, Cox BJ, Enns MW, Swinson RP. Psychometric properties of the 42-item and 21-item versions of the depression anxiety stress scales in clinical groups and a community sample. Psychol Assess 1998;10:176- 81.

13. Ogawa R, Seo E, Maeno T, Ito M, Sanuki $\mathrm{M}$, Maeno $\mathrm{T}$. The relationship between long working hours and depression among first- year residents in Japan. BMC Med Educ 2018;18:50.

14. Tan BY, Chew NW, Lee GK, Jing M, Goh Y, Yeo LL, et al. Psychological Impact of the COVID-19 Pandemic on Health Care Workers in Singapore. Ann Int Med 2020

15. Independent Coronavirus. NHS doctor kicked out by landlord because of Covid19

fears.

https://www.independent.co.uk/news/healt $\mathrm{h} /$ coronaviruslatest-nhs-doctor-evictedcovid-19-oxford-a9425166.html; 2020 (accessed on 2nd April, 2020)

16. Ministry of Health Singapore. Updates on COVID-19 (coronavirus disease 2019) local situation. Ministry of Health, Singapore. Accessed at www.moh.gov . sg/covid-19. 